\title{
A characterization of the quaternion group
}

To Professor Mirela Ştefănescu, at her 70th anniversary

\section{Marius Tărnăuceanu}

\begin{abstract}
The goal of this note is to give an elementary characterization of the well-known quaternion group $Q_{8}$ by using its subgroup lattice.
\end{abstract}

\section{Introduction}

One of the most famous finite groups is the quaternion group $Q_{8}$. This is usually defined as the subgroup of the general linear group $G L(2, \mathbb{C})$ generated by the matrices

$$
\mathbf{1}=\left(\begin{array}{ll}
1 & 0 \\
0 & 1
\end{array}\right), \quad \mathbf{i}=\left(\begin{array}{cc}
i & 0 \\
0 & -i
\end{array}\right), \mathbf{j}=\left(\begin{array}{cc}
0 & 1 \\
-1 & 0
\end{array}\right), \quad \mathbf{k}=\left(\begin{array}{cc}
0 & i \\
i & 0
\end{array}\right)
$$

Using matrix multiplication, we have $Q_{8}=\{ \pm \mathbf{1}, \pm \mathbf{i}, \pm \mathbf{j}, \pm \mathbf{k}\}$ and $\mathbf{i}^{2}=\mathbf{j}^{2}=$ $\mathbf{k}^{2}=-\mathbf{1}, \mathbf{i j}=-\mathbf{j i}=\mathbf{k}, \mathbf{j} \mathbf{k}=-\mathbf{k j}=\mathbf{i}, \mathbf{k i}=-\mathbf{i} \mathbf{k}=\mathbf{j}$. Moreover, $\mathbf{1}$ is the identity of $Q_{8}$ and $\mathbf{- 1}$ commutes with all elements of $Q_{8}$. Remark that $\mathbf{i}, \mathbf{j}, \mathbf{k}$ have order 4 and that any two of them generate the entire group. In this way, a presentation of $Q_{8}$ is

$$
Q_{8}=\left\langle a, b \mid a^{4}=1, a^{2}=b^{2}, b^{-1} a b=a^{-1}\right\rangle
$$

(take, for instance, $\mathbf{i}=a, \mathbf{j}=b$ and $\mathbf{k}=a b$ ). We also observe that the subgroup lattice $L\left(Q_{8}\right)$ consists of $Q_{8}$ itself and of the cyclic subgroups $\langle\mathbf{1}\rangle$, $\langle-\mathbf{1}\rangle,\langle\mathbf{i}\rangle,\langle\mathbf{j}\rangle,\langle\mathbf{k}\rangle$. It is well-known that $Q_{8}$ is a hamiltonian group, i.e. a non-abelian group all of whose subgroups are normal. More precisely

Key Words: Quaternion group, Subgroup lattice, Diamond lattice, $L$-free group, Almost $L$-free group.

2010 Mathematics Subject Classification: Primary 20D30; Secondary 20D60, 20E15.

Received: October, 2011.

Revised: January, 2012.

Accepted: February, 2012. 
$Q_{8}$ is the hamiltonian group with the smallest order.

Other basic properties of the subgroups of $Q_{8}$ are the following:

- excepting $Q_{8}$, they are cyclic;

$-\langle-\mathbf{1}\rangle$ is a breaking point in the poset of cyclic subgroups of $Q_{8}$, that is any cyclic subgroup of $Q_{8}$ either contains $\langle-\mathbf{1}\rangle$ or is contained in $\langle-\mathbf{1}\rangle$;

$-\langle\mathbf{i}\rangle,\langle\mathbf{j}\rangle$ and $\langle\mathbf{k}\rangle$ are irredundant, that is no one is contained in the union of the other two, and they determine a covering of $Q_{8}$, that is $Q_{8}=$ $\langle\mathbf{i}\rangle \cup\langle\mathbf{j}\rangle \cup\langle\mathbf{k}\rangle$.

These properties can be easily extended to some simple but very nice characterizations of $Q_{8}$ (see e.g. [7]), namely

$Q_{8}$ is the unique non-abelian p-group all of whose proper subgroups are cyclic,

$Q_{8}$ is the finite non-cyclic group with the smallest order whose poset of cyclic subgroups has a unique breaking point

and

$Q_{8}$ is the unique non-abelian group that can be covered by any three irredundant proper subgroups,

respectively.

The purpose of this note is to provide a new characterization of $Q_{8}$ by using another elementary property of $L\left(Q_{8}\right)$. We recall first a subgroup lattice concept introduced by Schmidt [3] (see also [4]). Given a lattice $L$, a group $G$ is said to be $L$-free if $L(G)$ has no sublattice isomorphic to $L$. Interesting results about $L$-free groups have been obtained for several particular lattices $L$, as the diamond lattice $M_{5}$ and the pentagon lattice $N_{5}$ (recall here only that a group is $M_{5}$-free if and only if it is locally cyclic, and $N_{5}$-free if and only if it is a modular group).

Clearly, for a finite group $G$ the above concept leads to the more general problem of counting the number of sublattices of $L(G)$ that are isomorphic to a certain lattice. Following this direction, our next definition is very natural.

Definition 1.1. Let $L$ be a lattice. A group $G$ is called almost $L$-free if its subgroup lattice $L(G)$ contains a unique sublattice isomorphic to $L$. 
Remark that both the Klein's group $\mathbb{Z}_{2} \times \mathbb{Z}_{2}$ and $Q_{8}$ are almost $M_{5}$-free (it is well-known that $L\left(\mathbb{Z}_{2} \times \mathbb{Z}_{2}\right) \cong M_{5}$, while for $Q_{8}$ the (unique) diamond is determined by the subgroups $\langle\mathbf{- 1}\rangle,\langle\mathbf{i}\rangle,\langle\mathbf{j}\rangle,\langle\mathbf{k}\rangle$ and $\left.Q_{8}\right)$. Our main theorem proves that these two groups exhaust all finite almost $M_{5}$-free groups.

Theorem 1.2. Let $G$ be a finite almost $M_{5}$-free group. Then either $G \cong$ $\mathbb{Z}_{2} \times \mathbb{Z}_{2}$ or $G \cong Q_{8}$.

In particular, we infer the following characterization of $Q_{8}$.

Corollary 1.3. $Q_{8}$ is the unique finite non-abelian almost $M_{5}$-free group.

Finally, we observe that there is no finite almost $N_{5}$-free group (indeed, if $G$ would be such a group, then the subgroups that form the pentagon of $L(G)$ must be normal; in other words, the normal subgroup lattice of $G$ would not be modular, a contradiction).

Most of our notation is standard and will usually not be repeated here. Basic notions and results on groups can be found in [1] and [5]. For subgroup lattice concepts we refer the reader to [2] and [6].

\section{Proof of the main theorem}

First of all, we prove our main theorem for $p$-groups.

Lemma 2.1. Let $G$ be a finite almost $M_{5}$-free p-group for some prime $p$. Then $p=2$ and we have either $G \cong \mathbb{Z}_{2} \times \mathbb{Z}_{2}$ or $G \cong Q_{8}$.

Proof. Let $M$ be a minimal normal subgroup of $G$.

If there is $N \in L(G)$ with $|N|=p$ and $N \neq M$, then $M N \in L(G)$ and $M N \cong \mathbb{Z}_{p} \times \mathbb{Z}_{p}$. Obviously, $\mathbb{Z}_{p} \times \mathbb{Z}_{p}$ has more than one diamond for $p \geq 3$. So, we have $p=2$ and we easily infer that $G \cong \mathbb{Z}_{2} \times \mathbb{Z}_{2}$.

If $M$ is the unique minimal subgroup of $G$, then by (4.4) of [5], II, $G$ is a generalized quaternion 2-group, that is there exists an integer $n \geq 3$ such that $G \cong Q_{2^{n}}$. If $n \geq 4$, then $G$ contains a subgroup $H \cong Q_{2^{n-1}}$ and therefore $G / \Phi(G) \cong \mathbb{Z}_{2} \times \mathbb{Z}_{2} \cong H / \Phi(H)$. This shows that $G$ has more than one diamond, a contradiction. Hence $n=3$ and $G \cong Q_{8}$, as desired.

We are now able to complete the proof of Theorem 1.2.

Proof of Theorem 1.2. We will proceed by induction on $|G|$. Let $H$ be the top of the unique diamond of $G$. We distinguish the following two cases. 
Case 1. $H=G$.

We infer that every proper subgroup of $G$ is $M_{5}$-free and therefore cyclic. Assume that $G$ is not a $p$-group. Then the Sylow subgroups of $G$ are cyclic. If all these subgroups would be normal, then $G$ would be the direct product of its cyclic Sylow subgroups and hence it would be cyclic, a contradiction. It follows that there is a prime $q$ such that $G$ has more than one Sylow $q$ subgroup. Let $S, T \in \operatorname{Syl}_{q}(G)$ with $S \neq T$. Since $S$ and $T$ are cyclic, $S \wedge T$ is normal in $S \vee T$ and the quotient $S \vee T / S \wedge T$ is not cyclic (because it contains two different Sylow $q$-subgroups). Hence $S \vee T=G$ and $G / S \wedge T$ is almost $M_{5}$-free. If $S \wedge T \neq 1$, then the inductive hypothesis would imply that $G / S \wedge T$ would be a 2 -group (isomorphic to $\mathbb{Z}_{2} \times \mathbb{Z}_{2}$ or to $Q_{8}$ ), contradicting the fact that it has two different Sylow $q$-subgroups. Thus $S \wedge T=1$. This shows that $\operatorname{Syl}_{q}(G) \cup\{1, G\}$ is a sublattice of $L(G)$. Since $G$ is almost $M_{5^{-}}$ free, one obtains $\left|S y l_{q}(G)\right|=3$. By Sylow's theorem we infer that $q=2$ and $\left|G: N_{G}(S)\right|=\left|S y l_{q}(G)\right|=3$. In this way, we can choose a 3 -element $x \in G \backslash N_{G}(S)$. It follows that $X=\langle x\rangle$ operates transitively on $\operatorname{Syl}_{q}(G)$. Then for every $Q \in \operatorname{Syl}_{q}(G)$, we have $Q \vee X \geq Q \vee Q^{x}=G$ and consequently $Q \vee X=G$. On the other hand, we obviously have $Q \wedge X=1$ because $Q$ and $X$ are of coprime orders. So $\{1, S, T, X, G\}$ is a second sublattice of $L(G)$ isomorphic to $M_{5}$, contradicting our hypothesis. Hence $G$ is a $p$-group and the conclusion follows from Lemma 2.1.

Case 2. $H \neq G$.

By the inductive hypothesis we have either $H \cong \mathbb{Z}_{2} \times \mathbb{Z}_{2}$ or $H \cong Q_{8}$. We also infer that $H$ is the unique Sylow 2-subgroup of $G$. Let $p$ be an odd prime dividing $|G|$ and $K$ be a subgroup of order $p$ of $G$. Then $H K$ is an almost $M_{5}$-free subgroup of $G$, which is not isomorphic to $\mathbb{Z}_{2} \times \mathbb{Z}_{2}$ or to $Q_{8}$. This shows that $H K=G$. Denote by $n_{p}$ the number of Sylow $p$-subgroups of $G$. If $n_{p}=1$, then either $G \cong \mathbb{Z}_{2} \times \mathbb{Z}_{2} \times \mathbb{Z}_{p}$ or $G \cong Q_{8} \times \mathbb{Z}_{p}$. It is clear that the subgroup lattices of these two direct products contain more than one diamond, contradicting our assumption. If $n_{p} \neq 1$, then $n_{p} \geq p+1 \geq 4$ and so we can choose two distinct Sylow $p$-subgroups $K_{1}$ and $K_{2}$. For $H \cong \mathbb{Z}_{2} \times \mathbb{Z}_{2}$ one obtains that $L_{1}=\left\{1, H, K_{1}, K_{2}, G\right\}$ forms a diamond of $L(G)$, which is different from $L(H)$, a contradiction. For $H \cong Q_{8}$ the same thing can be said by applying a similar argument to the quotient $G / H_{0}$, where $H_{0}$ is the (unique) subgroup of order 2 of $G$. This completes the proof.

We end our note by indicating three open problems concerning this topic.

Problem 2.2. Describe the (almost) $L$-free groups, where $L$ is a lattice different from $M_{5}$ and $N_{5}$. 
Problem 2.3 Determine explicitly the number of sublattices isomorphic to a given lattice that are contained in the subgroup lattices of some important classes of finite groups.

Problem 2.4. Extend the concepts of $L$-free group and almost $L$-free group to other remarkable posets of subgroups of a group (e.g. what can be said about a group whose normal subgroup lattice/poset of cyclic subgroups contains a certain number of sublattices isomorphic to a given lattice?).

Acknowledgements. The author is grateful to the reviewer for its remarks which improve the previous version of the paper.

\section{References}

[1] Isaacs, I.M., Finite group theory, Amer. Math. Soc., Providence, R.I., 2008.

[2] Schmidt, R., Subgroup lattices of groups, de Gruyter Expositions in Mathematics 14, de Gruyter, Berlin, 1994.

[3] Schmidt, R., L-free groups, Illinois J. Math. 47 (2003), 515-528.

[4] Schmidt, R., L L10-free groups, J. Group Theory 10 (2007), 613-631.

[5] Suzuki, M., Group theory, I, II, Springer Verlag, Berlin, 1982, 1986.

[6] Tărnăuceanu, M., Groups determined by posets of subgroups, Ed. Matrix Rom, Bucureşti, 2006.

[7] Tărnăuceanu, M., A characterization of generalized quaternion 2groups, C. R. Math. Acad. Sci. Paris 348 (2010), 731-733, doi: 10.1016/j.crma.2010.06.016.

Marius Tărnăuceanu

Faculty of Mathematics

"Al.I. Cuza" University

Iaşi, Romania

E-mail: tarnauc@uaic.ro 